\title{
Aging and Technology
}

\section{Richard Ferraro}

Professor Department of Psychology University North Dakota USA

“Corresponding author: Richard Ferraro, Department of Psychology, University North Dakota, USA, Tel: + (701) 777-2414; E-mail: f.richard.ferraro@email.und.edu

Received date: December 15, 2015; Accepted date: December 17, 2015; Published date: December 24, 2015

Copyright: (c) 2015 Ferraro R, et al. This is an open-access article distributed under the terms of the Creative Commons Attribution License, which permits unrestricted use, distribution, and reproduction in any medium, provided the original author and source are credited.

\section{Editorial}

In a recent article (Ferraro, Wunderlich, Wyrobek, \& Weivoda, 2014) [1], my co-authors and I observed some interesting relationships between age and texting and iPod dependence. We wanted to see if texting and iPod dependence were related to each other (they are) but also included a wide age range of subjects, primarily due to the fact that we were employing MTurk (Buhrmester, Kwang, \& Gosling, 2011) [2]. Across all four of the texting and iPod dependence scales (Emotional Reaction, Excessive Use, Relationship Disruption, Psychological/Behavioral Symptoms), all relationships with the variable Age were negative (r's ranged from -0.06 to -0.45 ) and all but one was significant $(p s<0.01)$. Thus, as one ages, it appears that dependence on these ubiquitous social media devices appears to decline. To our knowledge, this is one of the first observations of how dependence on some social media devices is impacted by age and it offers up a number of interesting perspectives on age and technology. It is known that there have been steady increases in how age and technology are associated (Czaja, Charness, Fisk, Hertzog, Nair, Rogers, et al, 2006) [3], and this observation adds to that everincreasing literature.

Our results, in combination with observations from others, raise the interesting issue that while ageing is associated with more technology use, such usage is also somewhat tempered by the fact that, unlike younger individuals, older adults show less dependence across a wide range of such dependencies. Such decreases in dependency suggests that older adults embrace technology and social media, but do not get so involved in it that it results in daily life disruption, emotional and psychological issues. Rather, older adults pace themselves with newer technologies, participating but not falling victim to these technologies. Such an attitude would result in a better transition of older adults continuing to use technology, using it effectively, and not be afraid of it. We see our observations as a starting point for the investigation of the many advances in technology and social media and their profound effect on older adults. These results are also a step in the right direction when attempting to downplay the many stereotypes about age and technology (specifically that older adults are afraid of technology). They are clearly not afraid. Rather, they want to know as much about the impact technology may have on them before they fully embrace it.

\section{References}

1. Ferraro FR, Wunderlich L, Wyrobek K, Weivoda L (2014) Does iPod dependence relate to text-message dependence? Psychological Record 64: 499-502.

2. Buhmester M, Kwang T, Gosling SD (2011) Amazon's Mechanical Turk: A new source of inexpensive, yet high-quality data? Psychological Science 6: 3-5.

3. Czaja SJ, Charness N, Fisk AD, Hertzog C, Nair SN, Rogers WA, et al. (2006) Factors predicting the use of technology: Findings from the Center for Research and Education on Aging and Technology. Psychology \& Aging 21: 333-353. 